\title{
Large cell neuroendocrine carcinoma of the uterine cervix
}

\author{
Amir Habeeb, ${ }^{1}$ Hany Habeeb ${ }^{2}$
}

${ }^{1}$ Department of Obstetrics and Gynaecology, University College London, London, UK ${ }^{2}$ Department of Obstetrics and Gynaecology, Medway Maritime Hospital, Gillingham, UK

Correspondence to Amir Habeeb

amirhabeeb@hotmail.co.uk

Accepted 24 December 2018

Check for updates

(c) BMJ Publishing Group Limited 2019. No commercial re-use. See rights and permissions. Published by BMJ.

To cite: Habeeb A, Habeeb H. BMJ Case Rep 2019;12:e225880. doi:10.1136/bcr-2018225880

\section{SUMMARY}

We report a case of large cell neuroendocrine carcinoma of the uterine cervix. A 33-year-old woman presented with a 4-month history of irregular vaginal bleeding and suspicious cervix. Transvaginal ultrasound showed a $3 \times 3.8 \mathrm{~cm}$ cervical mass with a marked increase in the blood flow. MRI pelvis showed an exophytic tumour with left external iliac lymph node metastasis. Immunohistochemistry of the tumour cells showed strong positivity for the neuroendocrine markers synaptophysin and a very high Ki67 proliferation index. A diagnosis of high-grade large cell neuroendocrine carcinoma of the uterine cervix was made with FIGO stage IIA2. She was treated with chemotherapy and palliative radiotherapy but died 21 months after presenting. Neuroendocrine tumour of the uterine cervix is an extremely aggressive cancer with the late presentation - the need for a more rigorous treatment protocol as well as potential screening methods could improve outcomes for these patients.

\section{BACKGROUND}

Neuroendocrine tumours (NETs) are incredibly aggressive diagnoses and although they more commonly involve the gastroenteropancreatic tract and lung, they can rarely affect other tissues such as the uterine cervix accounting for only $0.5 \%-1 \%$ of all malignant tumours of the uterine cervix. ${ }^{12}$ The tumours have features of both the endocrine and nervous systems. Their differentiation in neoplastic cells is characterised by peptide hormone or bioamine secretion into the bloodstream. ${ }^{3}$ In addition to this, some of the features pertaining to these NETs are unique to the organ of origin.

Several different classifications have been developed for staging and grading NETs on the basis of differentiation relative to their neighbouring non-neoplastic cells. In general, tumours that show poor differentiation are considered high grade while well-differentiated cancers are suggestive of a lower grade and therefore less likely to be aggressive. When comparing low-grade NETs to high-grade NETs, it is apparent how the two have different clinical presentations as well as pathological features and molecular interactions which consequently affects their responses to therapy and overall prognosis. This may be stemmed from the fact that low-grade NETs typically arise from neuroendocrine neoplastic syndromes of which multiple endocrine neoplasia (MEN1) or von Hippel Lindau syndrome are key examples and tend to have an idle progression. ${ }^{4}$ When looking at the pathology, low-grade NETs show similar architecture and cell features to non-neoplastic neuroendocrine cells which manifests itself in a trabecular growth pattern with characteristic stippled chromatin. This is in addition to the neurosecretory proteins being present such as chromogranin A and synaptophysin as well as CD56 which is a cell marker-these allow for effective use of immunohistochemistry in their histological diagnosis. On the other hand, the highgrade NETs have fewer features associated with normal neuroendocrine cells with limited expression of these markers with a positive stain for $\mathrm{p} 16$ indicative of the association with oncogenic human papilloma viruses. ${ }^{4}$ The tumours can be subdivided into small and large cell type or carcinoid/atypical carcinoid. ${ }^{5}$

It is suggested that NETs of the uterine cervix originate from the pre-existing neuroendocrine cells in the epithelium. ${ }^{4}$ An alternative explanation reveals the possibility of reserve cells in the cervix themselves undergoing neuroendocrine metaplasia and even endocrine cell hyperplasia in normal endocervical epithelium next to an existing NET of the uterine cervix observed by Chan et al. ${ }^{6}$ Uterine cervix NETs still pose a diagnostic and therapeutic challenge for gynae-oncologists leading to the overall poor prognosis.

\section{CASE PRESENTATION}

A 33-year-old woman presented to the gynaecology clinic having been referred under the 2 -week wait referral for a 4-month history of irregular per vaginal bleeding that was progressively getting worse and a necrotic mass replacing normal cervical tissue seen on speculum examination. She had two cervical smears in August 2009 and August 2012 which were both completely normal. She had left for Sri Lanka after delivery to visit family and started experiencing intermittent bleeding and spotting 2 months later but did not seek medical attention until 3 months after the symptoms started. She saw a gynaecologist abroad who did a speculum examination. He explained to her the concerning nature of her symptoms and took a biopsy but there is no formal correspondence from them. She returned to the UK before the biopsy results and was seen immediately by a general practitioner who referred her and she was seen 10 days later by a gynaecologist. She did not have any medical illnesses and was not on any medication. On examination, she was fit and healthy with an excellent performance status. On speculum examination, there was a marked 


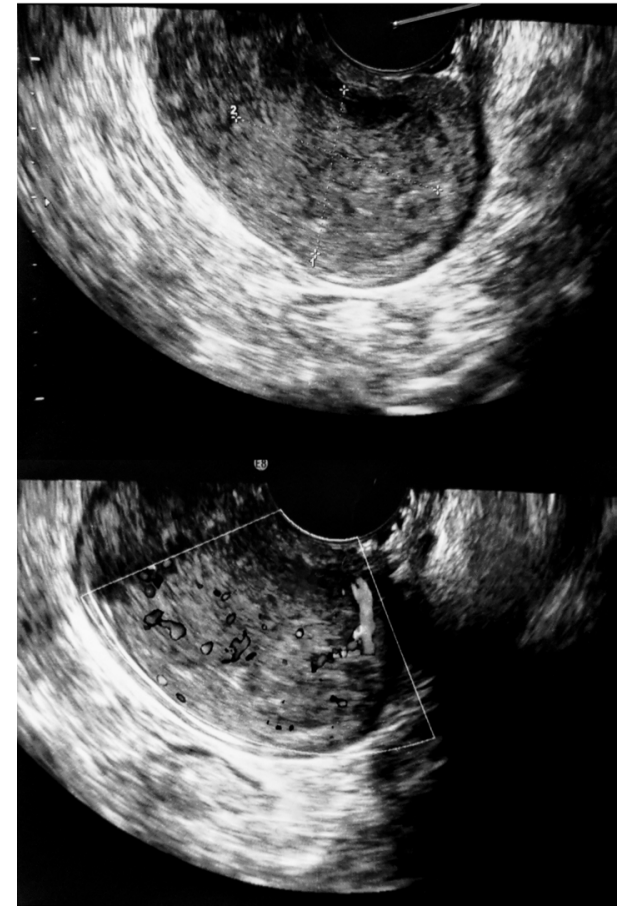

Figure 1 An ultrasound scan showing a $3 \times 3.8 \mathrm{~cm}$ cervical mass (top) with a marked increase in blood flow (bottom).

irregularity of the anterior cervical lip with surface ulceration. The clinical impression was that of invasive cervical cancer. Two punch biopsies were taken and sent for urgent histology. A set of blood samples were requested and sent for full blood count, clotting screen, urea and electrolytes and liver function tests which were all normal. Also, an urgent CT chest/abdomen/pelvis and MRI of the pelvis were requested.

\section{INVESTIGATIONS}

A transvaginal ultrasound scan revealed a large cervical mass measuring $3 \times 3.8 \mathrm{~cm}$ with a marked increase in the blood flow when the colour Doppler was applied (figure 1).

CT (figure 2) showed no significant mediastinal, hilar, axillary or supraclavicular adenopathy. There was no soft tissue parenchymal lung lesion. There was a focal thickening of the posterior pleura in the right posterior diaphragmatic recess but it was smoothly outlined and of benign appearance. There was no change in the adjacent ribs and no overall abnormal enhancement. There were no focal lesions in the liver and gallbladder, common bile duct and pancreas were all normal. The spleen was of normal size with no focal lesions and the adrenals appeared

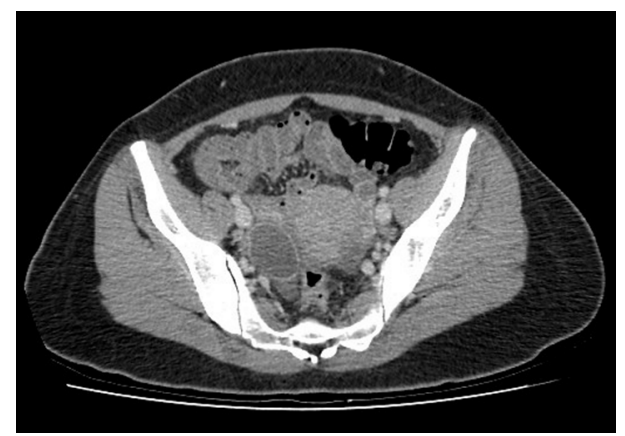

Figure 2 A CT pelvis with contrast showing an enlarged isodense cervix.

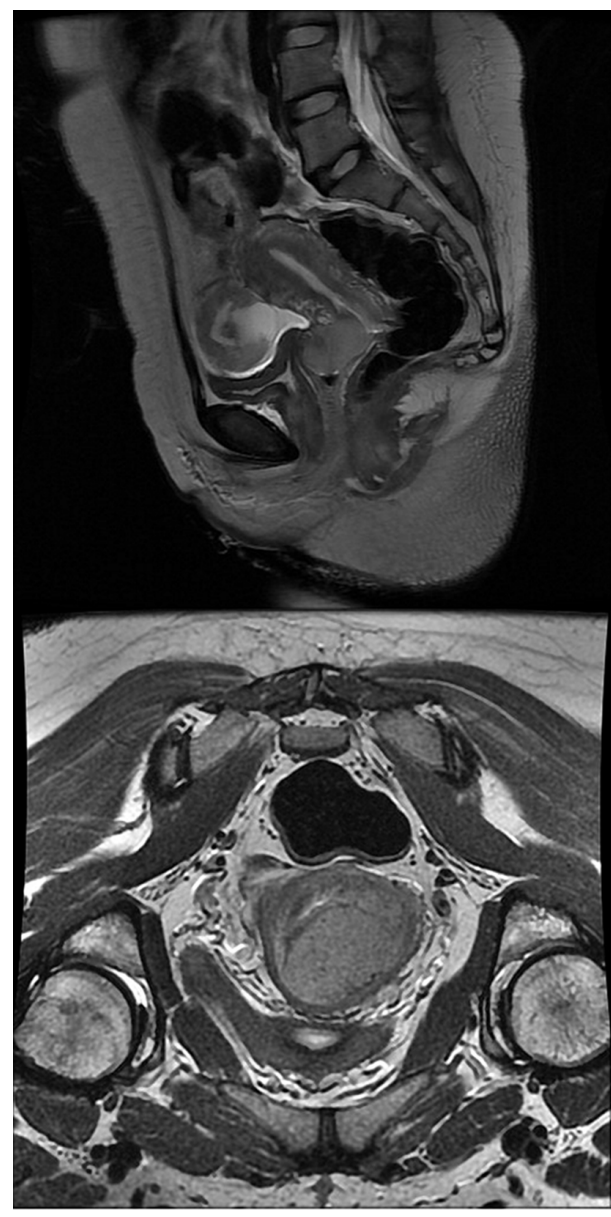

Figure 3 A T2-weighted MRI showing an exophytic tumour filling the upper vagina.

normal. Kidneys were unobstructed. Urinary bladder adequately filled and appeared normal. The uterus appeared normal although an enlarged isodense cervix was noted. The fat planes between the cervix and surrounding structures were preserved. There was a right adnexal cyst noted measuring $4 \times 3 \mathrm{~cm}$ and a $2 \mathrm{~cm}$ soft tissue noted near the left external iliac vein which could possibly be an enlarged/metastatic lymph node. There was no retroperitoneal, inguinal or mesenteric adenopathy. There was a trace of fluid in the pelvis around the uterus but otherwise no free fluid the abdomen. Peritoneal and mesenteric surfaces were grossly unremarkable. Unprepared colon appeared normal. There were no lytic or sclerotic bone lesions.

MRI (figure 3) of the pelvis showed an exophytic tumour filling the upper vagina with a tiny trace of free fluid within the pelvis and nodal/extranodal involvement. There was enlargement of the nodes in the left obturator fossa with a deposit in the left ovary consistent with a corpus luteal cyst. PET scan revealed a solitary malignant node in the external iliac lymph nodes. Radiology FIGO stage was given as IIA2.

Histopathology revealed fibrotic, chronically inflamed stroma infiltrated by a poorly differentiated malignancy characterised by nests of large, pleomorphic cells with prominent nucleoli, a moderate amount of cytoplasm, frequent mitoses and apoptosis. Immunohistochemistry of the tumour cells showed strong positivity for the neuroendocrine markers synaptophysin and CD56 and focal positivity for chromogranin. There is positivity for p16, CAM5.3 and a very high Ki67 proliferation index of nearly $100 \%$. Immunostaining for the squamous markers CK5/6 
was negative. Lymphovascular channel invasion was not seen. In conclusion, the biopsy revealed high-grade large cell neuroendocrine carcinoma with a FIGO stage of at least IB1 (figure 4).

\section{OUTCOME AND FOLLOW-UP}

She was started on a combination of cisplatin/etoposide chemotherapy immediately and later underwent palliative pelvic radiotherapy but sadly died 21 months after presenting to the gynaecology clinic.

\section{DISCUSSION}

NETs of the uterine cervix are very rare diagnoses with a poor clinical outcome due to the aggressive nature of the disease and early lymph node involvement with $65 \%$ of patients with large cell NETs of the uterine cervix dying within 3 years of diagnosis. ${ }^{1}$ It serves as a challenge both diagnostically and therapeutically for gynae-oncologists. ${ }^{7}$ With how uncommon it is, the aetiology and consequently predisposing risk factors are not well understood but there are certain demographics that seem more likely to have the diagnosis and thus offer potential for screening in the future as early diagnosis could improve outcomes. One study looked at the mean age of diagnosis and compared it with that of the more common squamous cell carcinoma of the cervix. ${ }^{8}$ McCusker $e t$ al showed the mean age was 49 in NETs (239 cases) versus 52 (18 453 cases) $(p<0.01)$. The obvious discrepancy in sample sizes does pose an issue but with the rarity of the diagnosis of NETs of the cervix, this is to be expected and statistical significance was still shown. Comparing ethnicities, it was apparent that a higher proportion of Asian women had NETs versus squamous cell carcinoma ( $10 \%$ vs $6 \%)(p=0.05)$. The patient we reported on was also Asian and perhaps a demographic of younger/Asian women could be proposed for screening. HPV and smoking are well-documented risk factors for developing the more common types of cervical cancer but their role in NETs of the uterine cervix are poorly understood. However, there might be an association between HPV-18 and small cell NETs of the uterine cervix. ${ }^{9}$ Unfortunately, the nature of squamous cell carcinoma of the uterine cervix means that there is a preinvasive stage in its clinical course while no such phase has been shown to exist making screening and even diagnosis an issue. ${ }^{4}$

Clinically, the common symptoms of presentation are no different to those of other more common types of cervical cancer but are very dependent on the time of presentation with regards to the stage of the disease. Abnormal vaginal bleeding as well as postcoital spotting are suggestive symptoms with some patients

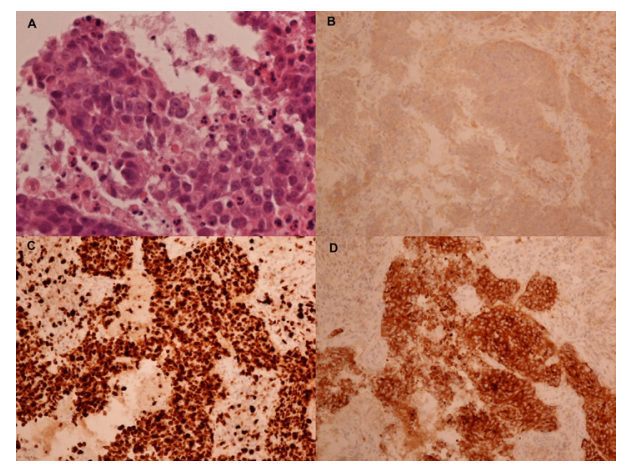

Figure 4 Histopathology slides with immunochemistry for the uterine cervix biopsies. (A) H\&E stain $\times 400$. (B) p63 negative (squamous cell marker). (C) Ki67 proliferation index positive. (D) Synaptophysin (neuroendocrine marker) positive. presenting with pelvic pain and/or dysuria. ${ }^{4}{ }^{6}$ With the high frequency of late presentation in these patients due to the aggressive nature of the disease, a lot of patients will have symptoms of advanced metastatic spread. ${ }^{6}$ Despite the neuroendocrine origin of these tumours, the expected carcinoid syndrome is actually very uncommon. It is hard to distinguish whether some symptoms are related to carcinoid syndrome or an oestrogen/progesterone imbalance in these patients although it is very possible that micrometastases to organs in the body in proximity to the systemic circulation such as the liver could then lead to carcinoid syndrome like symptoms. ${ }^{10}$ On gynaecological examination, the cervical neoplastic lesions detected tend to be large with an appearance described as barrel-shaped. ${ }^{6}$ Smears have not been shown to be effective in the workup and this woman had completely normal smears leading up to her diagnosis. Immunohistochemistry for NET markers aid the histopathological diagnosis and remain the gold standard. ${ }^{4}$

It is very apparent that delayed diagnosis in such a rapidly progressive cancer is something that needs to be avoided for better outcomes. Despite education around national screening for cervical cancer, it can be hard for patients to distinguish between what is normal and abnormal bleeding which should be considered in this case where the patient went 3 months before seeking medical attention for her symptoms. Although she was referred appropriately along the 2 -week wait referral system in the UK perhaps amendments can be made for women with such obvious pathology to be very urgently referred with the aim of starting treatment earlier.

There is no standard protocol as to how to treat large NETs of the uterine cervix. The multimodality approach including radical hysterectomy, systemic chemotherapy and radiotherapy is largely extrapolated from cases of lung NETs. ${ }^{4}$ In locally advanced disease, the recommended approach is a combination of chemotherapy with cisplatin and etoposide regimen followed by radiotherapy.

A method of screening in high-risk groups may serve a beneficial way of improving outcomes in a diagnosis that presents very late in its disease progression. Focusing on targeting the molecular and genetic aspects of NETs could serve beneficial in the future. ${ }^{4}$

\section{Learning points}

- Consider neuroendocrine carcinoma in a younger woman who presents with abnormal vaginal bleeding akin to other gynaecological cancers but in the background of an aggressive disease and even in the presence of normal smear history.

- Immunohistochemistry still remains the gold standard for diagnosis - manipulating this for screening reserve cells in at high risk groups may help improve outcomes for these women.

- A multimodality approach of radical hysterectomy, chemotherapy and radiotherapy is still the mainstay but this is extrapolated from cases of lung neuroendocrine cancer.

Contributors $\mathrm{AH}$ : wrote all parts of the case report with help of the patient notes. $\mathrm{HH}$ : saw the patient who presented in the clinic and managed her throughout.

Funding The authors have not declared a specific grant for this research from any funding agency in the public, commercial or not-for-profit sectors.

Competing interests None declared.

Patient consent for publication Not required.

Provenance and peer review Not commissioned; externally peer reviewed. 


\section{REFERENCES}

1 Gilks CB, Young RH, Gersell DJ, et al. Large cell neuroendocrine [corrected] carcinoma of the uterine cervix: a clinicopathologic study of 12 cases. Am J Surg Pathol 1997;21:905-14.

2 Huang L, Li C, Chen C, et al. Small Cell Carcinoma of the Uterine Cervix: a case report with emphasis on cytopathological findings. Tzu Chi Med J 2006;18:456-61.

3 Klimstra DS, Modlin IR, Coppola D, et al. The pathologic classification of neuroendocrine tumors: a review of nomenclature, grading, and staging systems. Pancreas 2010;39:707-12.

4 Gadducci A, Carinelli S, Aletti G. Neuroendrocrine tumors of the uterine cervix: a therapeutic challenge for gynecologic oncologists. Gynecol Oncol 2017;144:637-46.

5 Mannion C, Park WS, Man YG, et al. Endocrine tumors of the cervix: morphologic assessment, expression of human papillomavirus, and evaluation for loss of heterozygosity on 1p,3p, 11q, and 17p. Cancer 1998;83:1391-400.
6 Chan JK, Tsui WM, Tung SY, et al. Endocrine cell hyperplasia of the uterine cervix. A precursor of neuroendocrine carcinoma of the cervix? Am J Clin Pathol 1989;92:825-30.

7 Bermúdez A, Vighi S, García A, et al. Neuroendocrine cervical carcinoma: a diagnostic and therapeutic challenge. Gynecol Oncol 2001;82:32-9.

8 McCusker ME, Coté TR, Clegg LX, et al. Endocrine tumors of the uterine cervix: incidence, demographics, and survival with comparison to squamous cell carcinoma. Gynecol Oncol 2003;88:333-9.

9 Stoler MH, Mills SE, Gersell DJ, et al. Small-cell neuroendocrine carcinoma of the cervix. A human papillomavirus type 18-associated cancer. Am J Surg Pathol $1991 ; 15: 28-32$.

10 Stockdale AD, Leader M, Phillips $\mathrm{RH}$, et al. The carcinoid syndrome and multiple hormone secretion associated with a carcinoid tumour of the uterine cervix. Case report. Br J Obstet Gynaecol 1986;93:397-401.

Copyright 2018 BMJ Publishing Group. All rights reserved. For permission to reuse any of this content visit https://www.bmj.com/company/products-services/rights-and-licensing/permissions/

BMJ Case Report Fellows may re-use this article for personal use and teaching without any further permission.

Become a Fellow of BMJ Case Reports today and you can:

- Submit as many cases as you like

- Enjoy fast sympathetic peer review and rapid publication of accepted articles

- Access all the published articles

Re-use any of the published material for personal use and teaching without further permission

For information on Institutional Fellowships contact consortiasales@bmjgroup.com

Visit casereports.bmj.com for more articles like this and to become a Fellow 